\title{
Shift Work Type Circadian Rhythm Sleep Disorder
}

National Cancer Institute

\section{Source}

National Cancer Institute. Shift Work Type Circadian Rhythm Sleep Disorder. NCI

Thesaurus. Code C95075.

A subtype of circadian rhythm sleep disorder in which the individual exhibits a normal endogenous pattern of sleep and wakefulness, but this pattern comes into conflict with the desired pattern of sleep and wakefulness required by shift work. 